\title{
Microsurgical Flaps in Nipple Sparing Mastectomy: Surgical Techniques and Aesthetic Principles
}

\author{
Beina Azadgoli, MD'; Daniel J. Gould, MD, PhD'; Ketan M. Patel, MD*
}

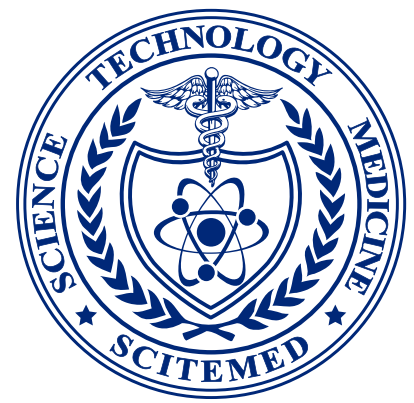

'Division of Plastic and Reconstructive Surgery, Keck School of Medicine, University of Southern California, Los Angeles, California, USA

\begin{abstract}
Nipple-sparing mastectomy (NSM) is gaining favor as surgeons become more comfortable with the surgery and as surgical indications expand. Autologous reconstruction is a popular method for breast restoration after mastectomy. In this article we describe various patient and breast-related factors that influence surgical outcomes while also addressing some techniques and principles for aesthetic microsurgical reconstruction.
\end{abstract}

\section{Introduction}

The focus of breast cancer treatment has evolved and now allows for more techniques geared towards sparing the breast envelope. In the past, the only option for mastectomies included excision of the nipple and adjacent tissue as an ellipse, which then required a process expansion or free tissue transfer with skin to compensate for that loss of breast skin and nipple. Studies comparing nipple-sparing mastectomy (NSM) to skin-sparing mastectomy (SSM) and modified radical mastectomy (MRM) have shown similar recurrence and survival rates across the three groups, confirming the overall safety of NSM in patients who are good candidates [1,2]. As such, NSM has gained favor as a surgical technique whenever it is oncologically possible to improve the overall quality of life for women following cancer treatment. NSM has allowed for total preservation of the native breast envelope and this, in turn, has allowed for improvements in aesthetic outcomes.

Improvements in mastectomy incisions and surgical techniques have led to the evolution of implant based and autologous reconstruction after NSM. In this article, we will focus on reconstructive principles after NSM and technical approaches. Microsurgical reconstruction after NSM can be performed through a variety of incisions [3], and teams must recognize that incision choice can affect the aesthetic result, the difficulty of the operation, and the perfusion and viability of the nipple postoperatively. Although necrosis rates following NSM generally range from $0-15 \%$, most cases can be managed conservatively, with little risk of total flap loss [4-8]. With developments in the understanding of breast surgery over time, the primary focus of reconstruction has shifted away from concerns about safety towards improvements in the aesthetic results.

When approaching autologous breast reconstruction, the plastic surgeon must consider several factors including tumor location, previous radiation and previous scars, as well as patient desires and breast characteristics. Technical refinements may be implemented to maximize aesthetic results and reduce the need for subsequent corrective procedures. Here, the authors attempt to address some of breast and patient factors as well as specific techniques for improving breast aesthetics following breast reconstruction.

\section{Aesthetic Principles}

\section{Breast Surgeon Experience}

One critical factor that determines outcomes of the procedure is the relationship between the plastic and breast or oncologic surgeon that is involved in the case. At centers where a plastic surgeon is not available, the oncologic surgeon is solely responsible for the mastectomy, and reconstruction is usually done in a delayed fashion. When possible, delayed-immediate techniques should be utilized to preserve breast skin as they have been shown to provide better outcomes [9]. While breast surgeons are familiar with oncoplastic techniques, they are not experienced with more advanced reconstructive procedures. At institutions where there is a multidisciplinary team including a plastic surgeon, there should be a discussion about the incision pattern and location or proximity of the tumor as well as the need, or potential need for radiation therapy. This type of multidisciplinary breast care may improve patient outcomes. Plastic surgeons must participate and advocate for aesthetically minded incision patterns and techniques. Surgeons who perform a high number of procedures together achieve better results, and thus the team dynamic can also greatly influence outcomes $[3,10]$.

\section{Breast Volume and Preoperative Planning}

Breast volume can be estimated prior to the case using several methods, including the vectra system. However, the breast volume can differ from the breast envelope capacity, and all patients are limited usually by the abdominal flap tissue in this surgery. In obese patients or in post-partum patients, there may be excessive flap skin or adiposity that needs to be trimmed to fit the breast envelope, but more commonly, the flap from the abdomen may fall short of the original breast glandular tissue. In these cases, one may consider a staged approach and may offer differed or immediate concomitant implant-based reconstruction. In immediate reconstruction, the implant can be placed behind the flap with an alloplastic implant material, either in a pre or retro pectoral space. In delayed implant augmentation, the implant can be placed through a traditional inframammary incision or through a peri areolar incision, below the flap.

\section{Incision Location}

The ideal incision should allow for a technically reasonable mastectomy and reconstruction while preserving adequate blood flow to the nipple areola complex (NAC) and reducing the likelihood of a visible scar as much as possible [11]. In certain cases, the nipple may be removed as a free nipple graft and incision choice may need to be tailored. Surgeons' nipple sparing mastectomy incisions influence surgical and aesthetic outcomes and should consider original breast shape in addition to tumor location. Importantly, the breast glandular tissue and the skin envelope should both be assessed, in that patients with preoperative ptosis may require mastopexy or skin reducing techniques, with consideration of different skin dimensions. Alternatively, patients with tight skin and dense breast tissue may require reduced flap volume to ensure flap viability 
early on. Patient preferences should be identified, and those seeking concomitant lift or volume augmentation must be counseled appropriately, though their wishes are not unattainable. Tumor location can affect the aesthetic outcome, as tumors on the inner quadrant have been associated with subpar cosmetic results [12]. More detail on incision will be discussed in the technical portion to follow.

\section{Ptosis}

Large, ptotic breasts introduce a different level of complexity that must be dealt with during the mastectomy. In addition to limiting the surgical exposure and being at higher risk for flap necrosis [13], they may also result in less favorable cosmetic results because of the larger skin envelope which may need to be tailored for the reconstruction. As a solution to this problem, various mastopexy-type incisions can be used in these patients [13]

Nipple preservation with skin tailoring places the patient at a significantly higher risk for skin and nipple necrosis as compared to single incision access. This may be due to the inherent poor vascularity of ptotic skin and nipple, or due to incision placement, which may reduce the subdermal plexus flow to the nipple skin and muscle. Primary nipple grafting is an option in combination with Wise-pattern or other multi-directional excisional patterns to allow for nipple repositioning. In skin preserving techniques that have robust subcutaneous tissue, nipple repositioning may be possible via the native blood supply. In these cases, fluorescent angiography may prove helpful to assess viability of the NAC.

In addition to these techniques to ensure viability, staged mastopexy incision patterns may be used to ease the perfusion burden to the lower pole of the breast [14]. In addition, de-epithelialization techniques can also prevent trifurcation junctions, which decrease chances of complications in the lower pole.

\section{Volume}

Assessment of breast volume is paramount during the preoperative stages of all breast surgery, including reconstruction. The original volume of the breast as well as the volume that must be excised significantly affect reconstructive outcomes, as the percentage of volume that must be removed is correlated with cosmetic outcomes and varies by quadrant [15]. Furthermore, the patients desired postoperative volume may help guide the aesthetic of the reconstructive surgeon. Mastectomies of greater volume are associated with higher rates of NAC necrosis [16]. Thus, one may argue that the ideal candidate for NSM is a patient with small/moderate-sized breasts with minimal ptosis. However, there are various techniques that can be applied to maximize results for women with larger and more ptotic breasts as well. Therefore, accurate prediction of breast volume can be helpful in determining the overall reconstructive plan as well as the need for simultaneous reduction and postoperative resection weight calculation.

\section{Symmetry in Unilateral vs. Bilateral}

Unilateral breast reconstruction can be quite challenging, as the reconstructed breast must match the healthy breast in shape, size, and projection. After unilateral mastectomy and reconstruction, surgery of the contralateral healthy breast, such as reduction, augmentation, or mastopexy, is often necessary for achieving symmetry.

Of the choices for post-mastectomy reconstruction, autologous reconstruction will generally have a better chance at achieving symmetry as compared to a normal contralateral side. When nipple-sparing mastectomy is chosen, subtle asymmetries can still occur, but are easily correctable at a staged revision surgery. In terms of patient satisfaction, patients who undergo autologous reconstruction have been shown to demonstrate higher satisfaction with unilateral reconstruction, suggesting that it may be more possible to achieve relative symmetry using autologous tissue [17]. Using 3D imaging, both TE-Implant and autologous reconstruction have been demonstrated to achieve symmetrical surgical results with the same number of operations. Thus, while breast symmetry is an important factor in preoperative planning of the reconstruction, it should not be the sole determining factor [18].

\section{Radiotherapy}

Post-mastectomy radiation therapy (PMRT) is commonly required in women with locally advanced breast cancer, particularly in high-risk patients with a tumor size of $\geq 5 \mathrm{~cm}$, positive axillary lymph nodes, or positive tumor margins [19]. In these patients, special consideration must be given to the type of reconstruction that is performed, as radiation can result in significant distortion of the breast tissue [20]. Although autologous reconstruction tolerates PMRT better than implant-based reconstruction, the incidence of contracture, fat necrosis, hyperpigmentation, and other flap-related complications remains high, leading to breast asymmetry and suboptimal aesthetic outcomes [21,22]. Although delayed reconstruction can be performed in these patients, aesthetic results are superior with immediate reconstruction due to the preservation of the skin envelope. As a solution to the need for revision procedures in patients who desire autologous reconstruction but need PMRT, delayed-immediate autologous reconstruction can be performed, during which a tissue expander is inserted to maintain the breast pocket following mastectomy and replaced with autologous tissue after radiation therapy has been completed [20]. Regardless, adjuvant therapy has a negative effect on surgical aesthetic outcomes [23].

\section{Techniques}

A wide variety of surgical incisions and techniques have been developed over the past several years in response to the recent surge in cases of nipple-sparing mastectomy [3]. Not one single approach has been demonstrated as superior however here we describe our senior author's preference.

\section{Vertical Incision}

In terms of the mastectomy, the vertical incision allows for excellent visualization of the breast tissue, while also providing access for the axillary dissection and breast reconstruction without the need for an additional scar in the upper pole of the breast (Figure 1A). Because the incision lies between and runs parallel to the medial and lateral mammary arteries, the chance of interrupting the blood supply to the flaps is reduced with this incision. With the vertical incision, it is possible to maintain viable skin flaps without the creation of a fragile T-junction, which is inevitable with other incisions. Additionally, it allows for relatively easy post-operative debridement if necessary, and further tightening of the incision can also be accomplished in the future without additional breast envelope incisions [24].

Better aesthetic outcomes are possible with this incision, as it results in more natural projection with improved nipple position [24]. The use of a vertical incision avoids flattening of the breast that occurs with horizontal incisions, particularly after the removal of a large amount of tissue. Although the vertical incision is great for small breasts, some skin envelope reduction is necessary with larger, more ptotic breasts [24] (Figure 2).

An additional benefit of the vertical incision is that it allows for greater correction of ptosis, and is particularly useful in those who need simultaneous contralateral breast mastopexy or reduction [8]. Breasts reconstructed with the vertical design have been shown to be aesthetically rated higher than their original appearance, particularly if done bilaterally [25]. One disadvantage to using this incision in a unilateral ptotic breast reconstruction is that the patient must typically also undergo a mastopexy in the unaffected breast for symmetry. Although vertical incisions are commonly used in mastopexy, the incision can be positioned higher or lower than in a mastopexy depending on the location of the tumor, and surgeons must be flexible in the design to allow for proper oncologic resection. Additionally, the vertical incision should be avoided in cases where the tumor is located far from the center of the breast [8].

Overall the advantages of the vertical incision include ease of dissection, precise inset, setup for revision, and camouflage of wound healing issues. Disadvantages include higher wound healing issues compared to a mid-breast incision and the vertical incision needs at least $6 \mathrm{~cm}$ length to accommodate flap inset. It is the preferred method for non-ptotic breasts (Table 1). 
A.

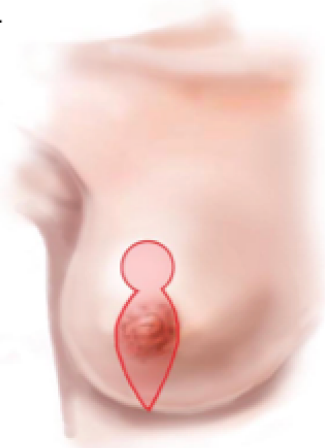

C.

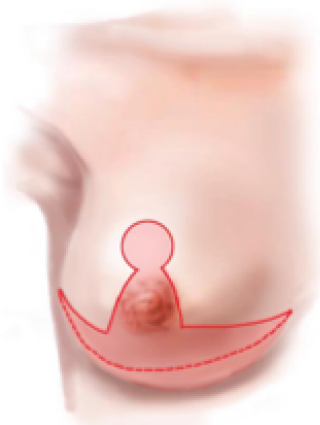

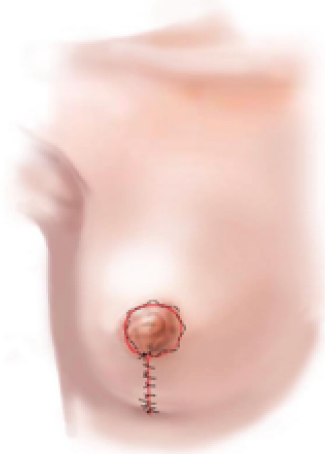

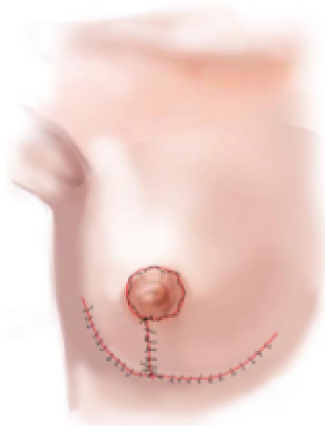

B.
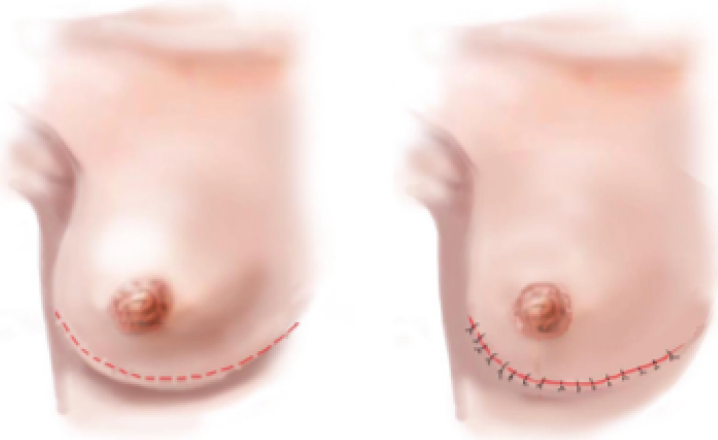

D.
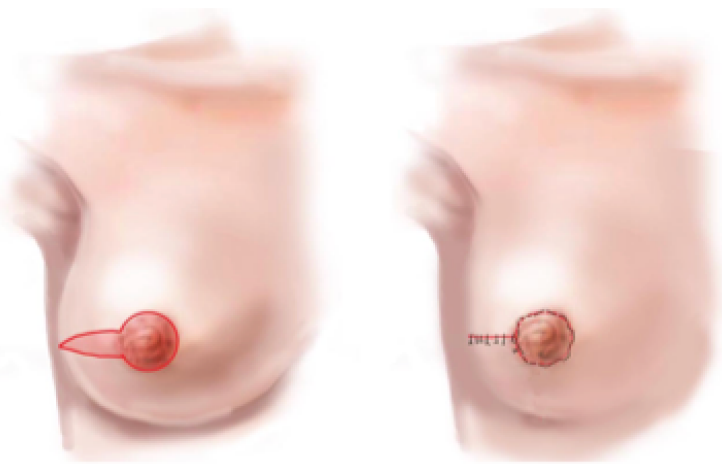

Figure 1. (A) Vertical incision. (B) Infra-mammary fold incision. (C) Modified-Wise pattern incision. (D) Radial incision.

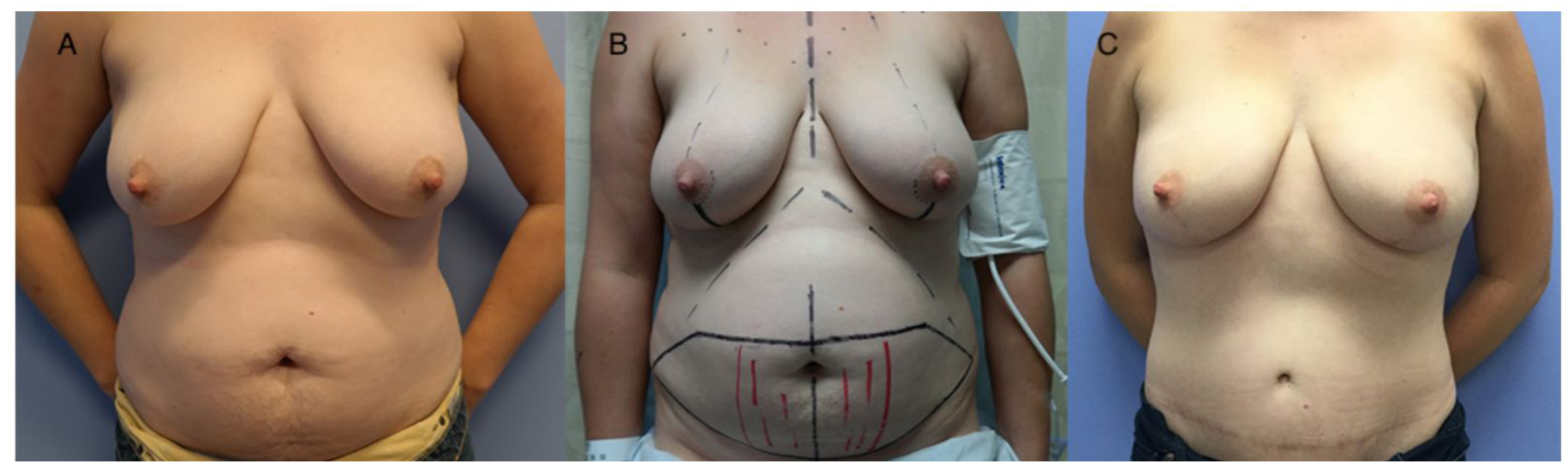

Figure 2. (A) Preoperative image of patient in need of bilateral prophylactic mastectomy. (B) Preoperative markings for vertical incision nipple-sparing mastectomy with single-perforator deep inferior epigastric perforators flaps. (C) Postoperative image of patient showing improved final contour and better defined superior pole.

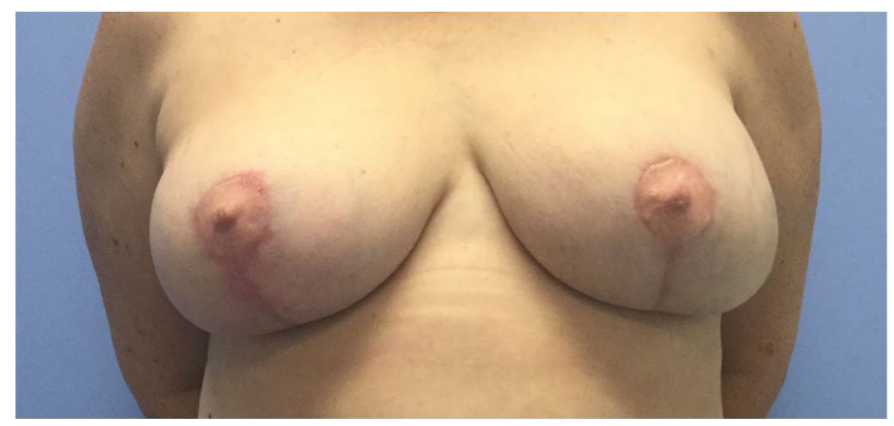

Table 1. Advantages and Disadvantages of Vertical Incision

\begin{tabular}{|l|l|}
\hline Advantages & Disadvantages \\
\hline $\begin{array}{l}\text { Breast surgeon ease of } \\
\text { dissection }\end{array}$ & $\begin{array}{l}\text { Higher wound healing issues } \\
\text { compared to mid-breast incision }\end{array}$ \\
\hline Precise inset & Need at least $6 \mathrm{~cm}$ to work \\
\hline Better setup for revision & \\
\hline Wound healing issues hidden & \\
\hline
\end{tabular}

Figure 3. Postoperative result of free nipple grafting. 

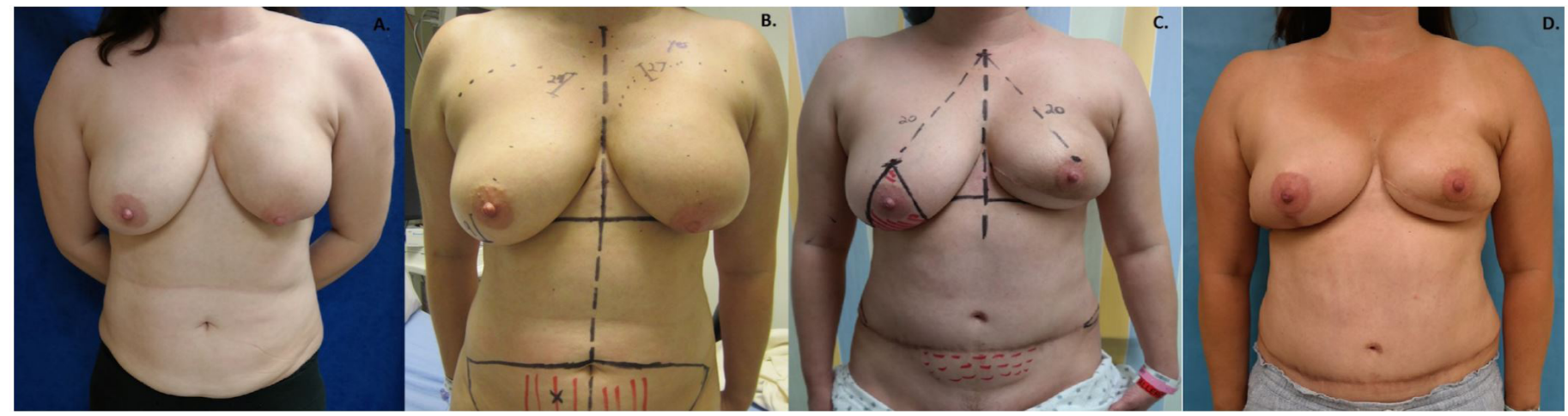

Figure 4. (A) Preoperative image of patient with recurrent cancer following breast-conserving therapy. (B) The breast mound and IMF on the contralateral side are marked for symmetry and the operative side IMF incision is drawn in parallel. (C) The planned nipple location is targeted based on the location of the ideal nipple on the native breast side; modified Wise-pattern markings for contralateral breast reduction for symmetry. (D) Patient postoperatively showing improved symmetry. IMF, inframammary fold incision.

A

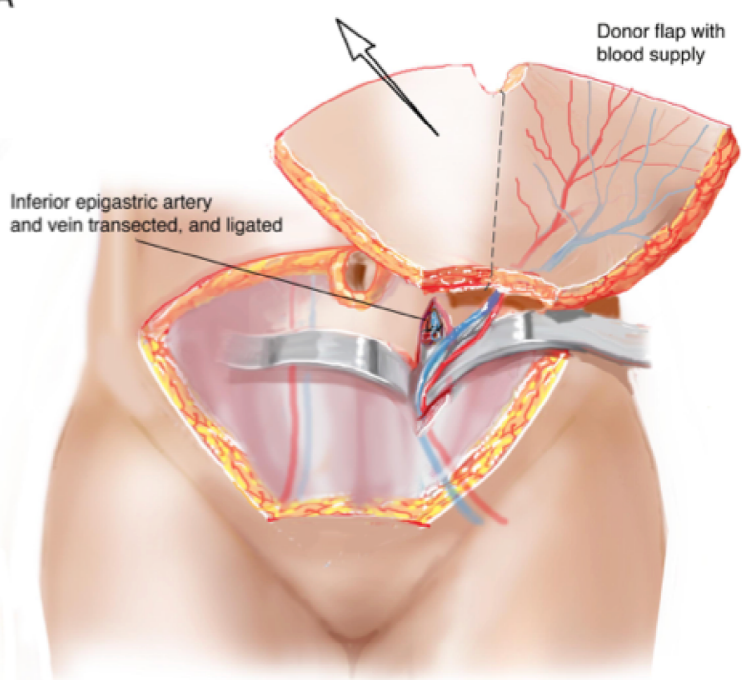

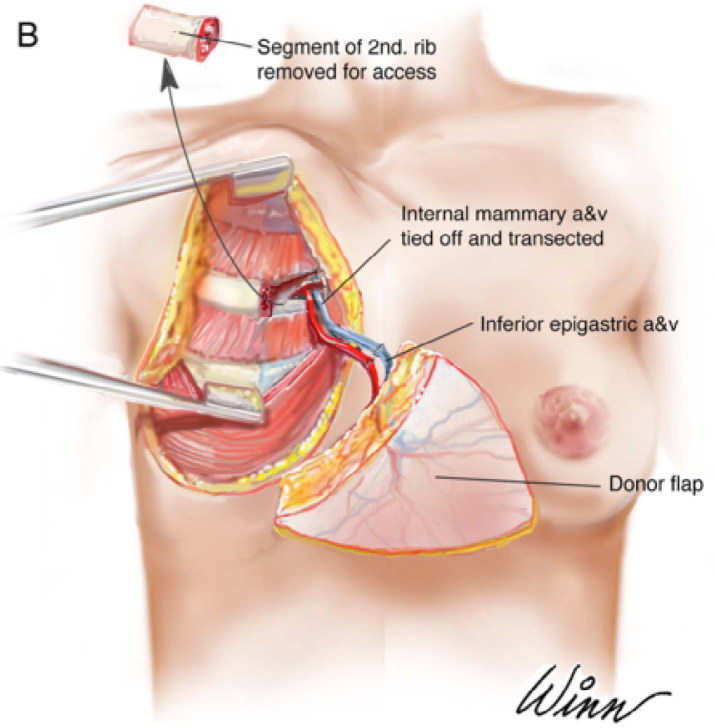

Figure 5. (A) Elevation of the deep inferior epigastric perforators flap with demonstration of inferior epigastric artery and vein. (B) Removal of second rib, inset of the donor flap, and anastomosis of inferior epigastric and internal mammary vessels.

\section{Inframammary Fold Incision}

The value of an inframammary fold incision (IMF) is of course widely recognized in aesthetic surgery and augmentation for providing good overall breast aesthetic outcomes. The IMF is hidden in the crease and avoidance of scars on the breast envelope allow for a natural, unoperated appearance to the nude breast (Figure 1B). The incision can be extended laterally for sentinel node excision or for additional resection of redundant flank tissue. Because of its relative simplicity, breast and cancer surgeons who are not trained in plastic surgery can also perform a mastectomy using this incision, yielding good cosmetic outcomes for a broader population [26]. Importantly, the IMF incision requires increased retraction and difficulty during separation of the gland from the NAC, and care must be taken to prevent destruction of its blood supply. One benefit of this incision is that the subdermal plexus all around the nipple can be preserved and a distant incision does not decrease or box nearby flow form the nascent skin. Though the overall necrosis rates with this incision are approximately $9 \%$, the risk of NAC necrosis is decreased if thicker flaps that leave most of the subcutaneous breast fat intact are used $[11,27]$. Additionally, access to the internal mammary or thoracodorsal pedicles can be challenging through this incision [13].

\section{Table 2. Advantages and Disadvantages of Modified Wise-Pattern} Incision

\begin{tabular}{|l|l|}
\hline Advantages & Disadvantages \\
\hline $\begin{array}{l}\text { Breast surgeon ease of dissection } \\
\text { Skin envelope reduction in both } \\
\text { vectors }\end{array}$ & Wound healing issues \\
\hline Nipple preservation & Nipple malposition with healing \\
\hline Allows for later revision & Deprojection of nipple \\
\hline
\end{tabular}

The inframammary fold incision results in excellent cosmesis and high rates of patient satisfaction [28]. Outcomes of breast reconstruction using the IMF incision based on NAC location, contour deformity, and breast symmetry, have been evaluated and $79 \%$ of patients have had good or very good results [27]. The incision leaves no visible scar on the anterior surface of the breast mound, hiding it within the natural crease of the breast, all while providing great exposure for tumor resection. Also, there is decreased risk of lateralization of the nipple with the use 
of this incision. It heals well with a low incidence of hypertrophic scarring [29], and it can be used for any future breast surgeries if the inferior skin is conserved.

This incision may be better for patients with smaller, less ptotic breasts, as those with larger, more ptotic breasts are at an increased risk for nipple malposition [29]. However, surgeons have reported successful results in patients with medium and large breasts with more ptosis [27].

One additional group of patients this incision is useful in is in patients with a previous history of breast conserving therapy with vertical skin loss or a tight lower pole. Inset of a new skin paddle from the flap in the lower breast pole will help correct tightness and will help improve the overall breast appearance. In general, this principal can be applied to patients with native breasts with tight lower poles as well.

\section{Modified Wise-Pattern}

Although the Wise-Pattern incision results in a high incidence of postoperative complications at the $\mathrm{T}$ junction where there are thin, angulated flaps coming together with great tension and inadequate blood flow $[25,30]$, the Modified Wise-Pattern attempts to resolve this problem while allowing for de-epithelialization of large amounts of excess tissue and good control of the resulting shape of the breast [25] (Figure 1C). Because the inferior flap has a stronger blood supply, it should be designed longer in high-risk patients to allow for a shorter superior flap, which is less perfused [30]. In larger breasts, primary free nipple grafts are harvested and placed back onto the flap skin. The remaining flap skin left can be placed in the shape of an areola or a halo. The halo allows for good suture fixation and allows for a rounder appearance to the nipple (Figure 3).

In cases where contralateral breast reduction or lift is desired, this incision results in good symmetry [30] and may be utilized for immediate contralateral breast surgery [31,32] or in a plan for delayed contralateral mastopexy (Figure 4) (Table 2). Overall, this pattern offers the surgeon a great deal of flexibility, with easy access to tumors in any location of the breast [33].

\section{Radial/Mid-Breast}

The radial incision is one of the most commonly performed techniques for NSM, representing approximately $46 \%$ of all incisions performed [11]. Historically the radial incision was developed from an adaptation of the skin sparing mastectomy techniques and accommodates most breast surgeons, as it allows the access they are familiar with from most training programs where skin-sparing mastectomy is common.

The radial incision provides excellent exposure of the breast, allowing for maximum tissue excision with good visualization and a more precise dissection (Figure 1D). In addition to providing adequate exposure of the axillary lymph nodes, the internal thoracic vessels are also easily visible for microsurgical anastomosis [34]. One downside is the incision abuts the nipple, and this peri-areolar type incision has been shown to increase necrosis of the nipple $[3,35,36]$, though this can be avoided if the incision is not carried more than 90 degrees around the areola.

While the surgeon has improved access for resection of the upper and outer quadrants of the breast with this incision, exposure of the upper medial breast becomes more difficult [7], [37]. Additionally, radial scar retraction can cause nipple lateralization postoperatively, although the lack of visible scars on the anterior breast mound does lead to improved aesthetic results [38].

This incision results in an overall nipple necrosis rate of $8.83 \%$ [11]. It is axial to the NAC periphery, and thus the skin-based blood supply to the nipple must be maintained [39]. Additional NAC perfusion is provided by the collateral blood supply from the intercostal arteries [39].

Over time, early centers that pioneered the NSM advocated for a variety of alternative incision choices [3] with the concept of preventing lateralization and preserving the breast envelope to prevent nipple necrosis, and also to conserve the breast skin for definitive reconstruction.

One important concept is that the biggest fear early in nipple sparing mastectomy was nipple necrosis or skin necrosis, primarily because early nipple sparing reconstruction was and still is largely performed with implant-based strategies. When using flap-based reconstruction, skin and nipple necrosis is less worrisome, because the flap is vascularized tissue and minor wound complications will not risk the reconstruction like in implant-based cases. Therefore, flap-based reconstruction allows for better creativity and safety of incision choices [40].

Similarly, ideal breast incisions for aesthetic breast surgery directly translate to ideal incisions for reconstructive nipple sparing mastectomy.

\section{Intra-Operative Techniques}

Preoperatively, computed tomographic angiography is often used to identify the dominant perforator prior to flap harvest [41]. During the surgical procedure, flaps are always harvested and placed on the contralateral breast, and they are usually rotated 100-110 degrees prior to inset (Figure 5). True perforator flaps allow for a longer pedicle, which allows for easier anastomoses and inset, giving greater degrees of freedom for rotation and adjustment. After completion of the microsurgical anastomosis on both sides, a small skin paddle is used for monitoring, and the rest of the flap skin is removed with a Colorado tip bovie, at the dermal junction. It is important to de-dermalize these flaps to prevent a palpable shelf of tissue, as thick abdominal skin can be palpable to the patient. At the edges of the reconstruction, the flap is rounded and sculpted with electrocautery to provide natural edges to the flap. The skin utilized for monitoring usually comes from the innermost border, near the midline incision which separates the flaps, and is usually no more than 1 by $2 \mathrm{~cm}$.

Sutures are placed to inset the flap on the chest wall. Two sutures are placed first medially to fasten the flap to the sternal pectoral fascial border, to prevent lateralization of the flap. Next, sutures are placed in the superior pole attached to the fascia just below the clavicle, preventing descent of the flap tissue and possibly helping to reduce the need for secondary fat grafting in the superior pole $[31,42]$. Finally, the lateral border is set with sutures which are placed between chest wall skin and the lateral-most aspect of the pectoral fascia, helping create the lateral anatomic definition to provide a natural appearing lateral breast mound. This lateral breast anatomy and border is paramount to the aesthetic appearance of a breast from the side in clothing.

Lastly, the inferior mammary fold is addressed by resetting the fold if it has been violated. Care must be taken to preserve a natural breast footprint, and as flaps become larger than the native tissue, the fold position must be addressed to preserve natural anatomy and nipple position.

Closure proceeds with standard deep dermal sutures and running subcuticular sutures with dermabond. Post-operative bras are placed on very large and ptotic reconstructions, while abdominal binders are placed for comfort on all patients, with care not to impinge on the lower breast or flap.

\section{Discussion}

The NSM was developed to improve aesthetic outcomes after the surgical treatment of breast cancer by allowing the reconstruction of a physiologically natural appearing breast. Breast reconstruction following NSM however involves an interdisciplinary approach between reconstructive plastic and breast surgeons, with considerations for tumor size and location, the need for radiotherapy, as well as breast size, shape, and degree of ptosis. Additionally, careful patient selection is necessary, particularly in smokers and those with high BMI. Although not all factors involved in the reconstruction can be controlled for by the plastic surgeon, there are certain technical refinements that can be made during surgery to help improve aesthetic outcomes and reduce the need for secondary procedures.

Autologous breast reconstruction following NSM is becoming more routinely performed, particularly in cases of implant reconstruction failure or in patients requiring radiation therapy. The aesthetic complications that must be considered include visible scars as well as breast contour deformities and nipple malposition secondary to scar contracture. Although these can be corrected at a future date with secondary surgery, fat grafting, or contouring, an appropriately planned incision can minimize this need and ultimately decrease patient morbidity. Furthermore, flap-based reconstruction may provide more flexibility and creativity as well as safety in nipple sparing reconstruction. Several studies have 
shown higher complication rates with the traditional peri-areolar approach $[3,35,36,43]$, which has led to the development of more creative approaches, some of which are described above. However, the incision of choice depends on the breast surgeon's experience and preference.

As there is no one best method of breast reconstruction, each patient should receive an individualized plan with an in-depth discussion regarding the various treatment options along with their risks and benefits. Although long-term scar contracture and effects of radiotherapy cannot be predicted or controlled for, deformities due to poor surgical planning should be avoided. The postoperative aesthetic result is a critical determinant of patient satisfaction following breast reconstruction, ultimately playing an important role in the psychological impact of breast cancer treatment.

\section{Conclusion}

The NSM-immediate free flap reconstruction is a favorable surgical option for the treatment of breast cancer in patients with no oncologic contraindications, and can also be considered as a prophylactic measure for high-risk patients. In addition to providing an oncologically safe treatment option, it also allows for excellent cosmetic outcomes with more physiological reconstructive results. Several factors must be taken into consideration by the plastic surgeon to achieve optimal outcomes. Each incision design has features that make it appropriate or inappropriate for a specific patient depending on patient and physician expectations. Surgeons should conduct a full assessment of their patients and select individual-specific incisions for the best aesthetic results. Further, new creative reconstructive techniques should be explored whenever feasible to expand the application of this procedure to a broader patient population.

\section{Article Information}

\section{*Correspondence: Ketan M. Patel, MD}

Division of Plastic and Reconstructive Surgery, Keck School of Medicine, University of Southern California, 1510 San Pablo Street, Suite 415, Los Angeles, CA, USA.

Email: ketan.patel@med.usc.edu

Received: May 01, 2018; Accepted: Jun. 14, 2018; Published: Jul. 23, 2018

\section{DOI: 10.24983/scitemed.imj.2018.00071}

Copyright (c) 2018 The Author(s). This is an open-access article distributed under the terms of the Creative Commons Attribution 4.0 International License (CC-BY).

\section{Funding: None}

\section{Conflict of Interest: None}

Informed Consent: Written informed consent was obtained from the patient for publication of this Case report and any accompanying images.

\section{Keywords}

Aesthetics; autologous breast reconstruction; breast reconstruction; DIEP flap; nipple-sparing mastectomy; oncoplastic breast surgery; technique.

\section{References}

1. Kim MS, Reece GP, Beahm EK, Miller MJ, Neely Atkinson E, Markey MK. Objective assessment of aesthetic outcomes of breast cancer treatment: measuring ptosis from clinical photographs. Comput Biol Med 2007;37(1):49-59.

2. Gerber B, Krause A, Dieterich M, Kundt G, Reimer T. The oncological safety of skin sparing mastectomy with conservation of the nipple-areola complex and autologous reconstruction: an extended follow-up study. Ann Surg 2009;249(3):461-468.
3. Gould DJ, Hunt KK, Liu J, et al. Impact of surgical techniques, biomaterials, and patient variables on rate of nipple necrosis after nipple-sparing mastectomy. Plast Reconstr Surg 2013;132(3).

4. Crowe JP, Kim JA, Yetman R, Banbury J, Patrick RJ, Baynes D. Nipple-sparing mastectomy: technique and results of 54 procedures. Arch Surg 2004;139(2):148-150.

5. Komorowski AL, Zanini V, Regolo L, Carolei A, Wysocki WM, Costa A. Necrotic complications after nipple- and areola-sparing mastectomy. World J Surg 2006;30(8):1410-1413.

6. Sacchini V, Pinotti JA, Barros AC, et al. Nipple-sparing mastectomy for breast cancer and risk reduction: oncologic or technical problem? J Am Coll Surg 2006;203(5):704-714.

7. Moyer HR, Ghazi B, Daniel JR, Gasgarth R, Carlson GW. Nipple-sparing mastectomy: technical aspects and aesthetic outcomes. Ann Plast Surg 2012;68(5):446-450.

8. Hunter JE, Malata CM. Refinements of the Lejour vertical mammaplasty skin pattern for skin-sparing mastectomy and immediate breast reconstruction. J Plast Reconstr Aesthetic Surg 2007;60(5):471481.

9. Albino FP, Patel KM, Smith JR, Nahabedian MY. Delayed versus delayed-immediate autologous breast reconstruction: a blinded evaluation of aesthetic outcomes. Arch Plast Surg 2014;41(3):264-270.

10. Gfrerer $L$, Mattos $D$, Mastroianni $M$, et al. Assessment of patient factors, surgeons, and surgeon teams in immediate implant-based breast reconstruction outcomes. Plast Reconstr Surg 2015;135(2):245e-252e.

11. Endara M, Chen D, Verma K, Nahabedian MY, Spear SL. Breast reconstruction following nipple-sparing mastectomy: a systematic review of the literature with pooled analysis. Plast Reconstr Surg 2013;132(5):1043-1054.

12. Cochrane RA, Valasiadou P, Wilson ARM, Al-Ghazal SK, Macmillan RD. Cosmesis and satisfaction after breast-conserving surgery correlates with the percentage of breast volume excised. Br J Surg 2003;90(12):1505-1509.

13. Sisco M, Yao KA. Nipple-sparing mastectomy: a contemporary perspective. J Surg Oncol 2016;113(8):883-890.

14. Liu TS, Crisera CA, Festekjian JH, Da Lio AL. Staged Wise-pattern skin excision for reconstruction of the large and ptotic breast. Plast Reconstr Surg 2010;126(6):1831-1839.

15. Pukancsik $D$, Kelemen $P$, Újhelyi $M$, et al. Objective decision making between conventional and oncoplastic breast-conserving surgery or mastectomy: an aesthetic and functional prospective cohort study. Eur J Surg Oncol 2017;43(2):303-310.

16. Cho J-W, Yoon E-S, You H-J, Kim H-S, Lee B-I, Park S-H. Nipple-areola complex necrosis after nipple-sparing mastectomy with immediate autologous breast reconstruction. Arch Plast Surg 2015;42(5):601. 607.

17. Craft RO, Colakoglu S, Curtis MS, et al. Patient satisfaction in unilateral and bilateral breast reconstruction [Outcomes article]. Plast Reconstr Surg 2011;127(4):1417-1424.

18. Cohen O, Small K, Lee C, Petruolo O, Karp N, Choi M. Is unilateral implant or autologous breast reconstruction better in obtaining breast symmetry? Breast J 2016;2(1):75-82.

19. Orecchia R. The use of postoperative radiation after nipple sparing mastectomy. Gland Surg 2016;5(1):63-68.

20. El-Sabawi B, Carey JN, Hagopian TM, Sbitany H, Patel KM. Radiation and breast reconstruction: algorithmic approach and evidence-based outcomes. J Surg Oncol 2016;113(8):906-912.

21. Patel KM, Albino F, Fan KL, Liao E, Nahabedian MY. Microvascular autologous breast reconstruction in the context of radiation therapy: comparing two reconstructive algorithms. Plast Reconstr Surg 2013:132(2):251-257.

22. Spear SL, Shuck J, Hannan L, Albino F, Patel KM. Evaluating long-term outcomes following nipple-sparing mastectomy and reconstruction in the irradiated breast. Plast Surg Nurs 2017;37(2):66-75. 
23. El-Sabawi B, Sosin M, Carey JN, Nahabedian MY, Patel KM. Breast reconstruction and adjuvant therapy: a systematic review of surgical outcomes. J Surg Oncol 2015;112(5): 458-464.

24. Becker H, Lind JG, Hopkins EG. Immediate implant-based prepectoral breast reconstruction using a vertical incision. Plast Reconstr Surg - Glob. Open, 2015;3(6):e412.

25. Bourne DA, Ahuja N, Gimbel ML. Analysis of the vertical mammaplasty design in skin-sparing mastectomy and immediate autologous reconstruction. J Plast Reconstr Aesthet Surg 2016;69(1):23-29.

26. Heiman UllmarkJ, Sahlin C, Hallberg H, Olofsson Bagge R. Breast-conserving surgery using an inframammary fold incision technique for breast cancer. J Plast Surg Hand Surg 2017;51(2):105-111.

27. Salibian AH, Harness JK, Mowlds DS. Inframammary approach to nipple-areola-sparing mastectomy. Plast Reconstr Surg 2013;132(5).

28. Dent BL, Small K, Swistel A, Talmor M. Nipple-areolar complex ischemia after nipple-sparing mastectomy with immediate implant-based reconstruction: risk factors and the success of conservative treatment. Aesthet Surg J 2014;34(4):560-570.

29. Huston TL, Small K, Swistel AJ, Dent BL, Talmor M. Nipple-sparing mastectomy via an inframammary fold incision for patients with scarring from prior lumpectomy. Ann Plast Surg 2015;74(6):652-657.

30. Skoll PJ, Hudson DA. Skin-sparing mastectomy using a modified wise pattern. Plast Reconstr Surg 2002;110(1):214-217.

31. Longo B, Laporta R, Pagnoni M, Santanelli di Pompeo F. A novel approach to achieve breast symmetry in a single-stage procedure. Plast Aesthet Res 2015;2:76-78.

32. Laporta R, Longo B, Sorotos M, Pagnoni M, Santanelli Di Pompeo F. One-stage DIEP flap breast reconstruction: algorithm for immediate contralateral symmetrization. Microsurgery 2016;36(1):7-19.

33. Chang MM, Huston T, Ascherman J, Rohde C. Oncoplastic breast reduction: maximizing aesthetics and surgical margins. Int J Surg Oncol $2012 ; 2012$.
34. Munhoz AM, Aldrighi CM, Montag E, et al. Clinical outcomes following nipple-areola-sparing mastectomy with immediate implant-based breast reconstruction: a 12-year experience with an analysis of patient and breast-related factors for complications. Breast Cancer Res Treat 2013;140(3):545-555

35. Laporta $\mathrm{R}$, Longo $\mathrm{B}$, Sorotos $\mathrm{M}$, et al. Breast reconstruction following nipple-sparing mastectomy: clinical outcomes and risk factors related complications. J Plast Surg Hand Surg 2017;51(6):427-435.

36. Rossi C, Mingozzi M, Curcio A, Buggi F, Folli S. Nipple areola complex sparing mastectomy. Gland Surg 2015;4(6):528-540.

37. Tegtmeier RE. The curved lateral incision technique for subcutaneous mastectomy. Ann Plast Surg 1981;6(3):194-202.

38. Salibian AH, Harness JK, Mowlds DS. Primary buttonhole mastopexy and nipple-sparing mastectomy: a preliminary report. Ann Plast Surg 2016;77(4):388-395.

39. Rawlani V, Fiuk J, Johnson SA, et al. The effect of incision choice on outcomes of nipple-sparing mastectomy reconstruction. Can J Plast Surg 2011;19(4):129-133.

40. Patel KM, Hill LM, Gatti ME, Nahabedian MY. Management of massive mastectomy skin flap necrosis following autologous breast reconstruction. Ann Plast Surg 2012;69(2):139-144.

41. Lee JS, Patel KM, Zou Z, Prince MR, Cohen El. Computerized tomographic and magnetic resonance angiography for perforator-based free flaps: technical considerations. Clinics in Plastic Surgery 2011;38(2):219-228.

42. Longo B, Laporta R, Sorotos M, Pagnoni M, Gentilucci M, Santanelli di Pompeo F. Total breast reconstruction using autologous fat grafting following nipple-sparing mastectomy in irradiated and non-irradiated patients. Aesthetic Plast Surg 2014;38(6):1101-1108.

43. Salgarello M, Visconti G, Barone-Adesi L. Nipple-sparing mastectomy with immediate implant reconstruction: Cosmetic outcomes and technical refinements. Plast Reconstr Surg 2010;126(5):1460-1471. 